\title{
Collocated Z-Axis Control of a High-Speed Nanopositioner for Video-Rate Atomic Force Microscopy
}

\author{
Yuen Kuan Yong, Member, IEEE, and S. O. Reza Moheimani, Fellow, IEEE
}

\begin{abstract}
A key hurdle to achieve video-rate atomic force microscopy (AFM) in constant-force contact mode is the inadequate bandwidth of the vertical feedback control loop. This paper describes techniques used to increase the vertical tracking bandwidth of a nanopositioner to a level that is sufficient for video-rate AFM. These techniques involve the combination of: a high-speed XYZ nanopositioner; a passive damping technique that cancels the inertial forces of the $Z$ actuator which in turns eliminates the low $20-\mathrm{kHz}$ vertical resonant mode of the nanopositioner; an active control technique that is used to augment damping to high vertical resonant modes at $60 \mathrm{kHz}$ and above. The implementation of these techniques allows a tenfold increase in the vertical tracking bandwidth, from 2.3 (without damping) to $28.1 \mathrm{kHz}$. This allows high-quality, video-rate AFM images to be captured at 10 frames/s without noticeable artifacts associated with vibrations and insufficient vertical tracking bandwidth.
\end{abstract}

Index Terms-Atomic force microscopy, constant-force, flexure, nanopositioning, video-rate.

\section{INTRODUCTION}

$\mathbf{T}$ $\mathrm{HE}$ atomic force microscope (AFM) has been a crucial instrument for studying and interrogating material surfaces on the sub-nanometer scale. The AFM produces 3-D topographical images of a sample by probing its surface with a sharp tip attached to the free end of a micro-cantilever. The sample is raster-scanned over a surface area. Interaction forces between the tip and the sample cause the micro-cantilever to deflect. The deflection measured at each scan point, which is a representation of the sample height, can then be plotted as a function of the tip's lateral position to generate the 3-D image of the surface. The versatility of AFMs and their unique abilities to investigate sample surfaces in air, vacuum and liquid environments have led to the development of a large number of operating modes, such as contact, semi-contact and non-contact modes [1], [2]. These developments have paved the way for substantial advancement in a variety of fields since the invention of AFM in the 1980's [3], including in biological and life sciences [4]-[6], semiconductor metrology and manufacturing [7]-[9], nanofabrication [10]-[12], and high-density data storage systems [13], [14].

Manuscript received August 12, 2014; revised November 20, 2014; accepted December 29, 2014. Date of publication January 20, 2015; date of current version March 6, 2015. This work was supported by the Australia Research Council.

The authors are with the School of Electrical Engineering and Computer Science, University of Newcastle, Callaghan, NSW 2308, Australia (e-mail: yuenkuan.yong@newcastle.edu.au; reza.moheimani@ newcastle.edu.au).

Color versions of one or more of the figures in this paper are available online at http://ieeexplore.ieee.org.

Digital Object Identifier 10.1109/TNANO.2015.2394327
The AFM's ultra-sharp tip (a few nanometer wide) provides it with $10^{6}$ times more magnification compared to that of conventional optical microscopes [15]. However, a commercial AFM may require a long time to acquire an image. For example, with a typical scan-rate of $1 \mathrm{~Hz}$, it takes more than 2 minutes to obtain a $128 \times 128$ pixel resolution image, which is too slow for high throughput applications such as in nanofabrication [11], [16] and imaging of fast biological processes [5], [17].

There are several factors that limit the scan speed of AFMs [1], [5], [18]-[21]. Many of these arise from limited bandwidth of certain components of the AFM system, including the resonance frequency of the micro-cantilever, the speed of the data acquisition system, the resonance frequency of the nanopositioner, and the bandwidth of the vertical feedback control loop that regulates the tip-sample interaction force. It is possible to achieve video-rate scanning by improving only the $\mathrm{X} / \mathrm{Y}$ tracking bandwidth through mechanical design and advanced control techniques, and to avoid the vertical feedback loop by operating in constant-height contact mode [22]-[24]. However, due to the lack of vertical feedback in constant-height mode, it is impossible to control the force acting on the sample during a scan. As a result, constant-force contact mode is a more suitable technique to avoid sample damage.

One approach to high-speed AFM is to separate the fast Zstage from the slow XY-stage [25]-[27]. This method avoids the vibrations induced by the fast $\mathrm{Z}$-stage to propagate through and excite the resonances of the XY-stage. Although this arrangement has the advantage of mechanically decoupling the $X Y$ and $\mathrm{Z}$ stages, it may (i) complicate the operation of the optical system that is used to measure the deflection of the micro-cantilever [25], and (ii) lead to a reduction of the mechanical bandwidth of the XY-stage [1], [28].

This paper focuses on achieving video-rate AFM scanning in constant-force contact mode using a high-speed XYZ nanopositioner. To achieve video-rate AFM, a flexure-based nanopositioner with high resonance frequencies in the $\mathrm{X}, \mathrm{Y}$ and $\mathrm{Z}$ axes and low cross-talk among the three axes is used to replace a commercial AFM XYZ scanner. The main challenge of using the XYZ nanopositioner in video-rate scanning is to simultaneously avoid vibrations in all three axes. This is because the fast movement of the vertical piezoelectric stack actuator generates impulsive forces that excite the XY resonant modes. Here, these impulsive forces are mechanically canceled (passively damped) by using a dual-mounted Z-stage configuration [23], [28], [29]. A pair of piezoelectric stack actuators are driven simultaneously with the same magnitude but in opposite 

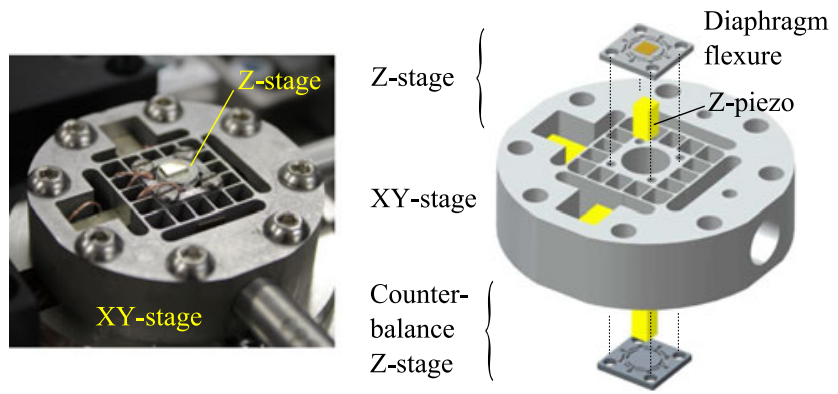

Fig. 1. Flexure-based XYZ nanopositioner.

direction to counterbalance the impulsive forces. As a result, the low resonant mode that lives at $20 \mathrm{kHz}$ is passively suppressed and the subsequent resonant modes which need to be controlled appear at $60 \mathrm{kHz}$ and above. To further increase the vertical bandwidth, active damping control technique is implemented to suppress these resonances. Here, the integral resonant control (IRC) technique is used to augment damping to the resonances. IRC has been used in damping multiple resonant modes of flexible structures with collocated sensors and actuators [30], [31]. In this work, the IRC technique is realized with a collocated force sensor and an integral controller [32]-[34]. The IRC method is simple to implement and it provides good robustness and guaranteed stability [30], [31], [35]. By combining both active and passive damping techniques, a $28.1 \mathrm{kHz}$ vertical tracking bandwidth is achieved. With this bandwidth, high-quality video-rate AFM images in constant-force contact mode are successfully recorded at 10 frames/s.

The remainder of the paper proceeds as follows. Section II describes the AFM nanopositioning system. The design and implementation of the passive and active damping control techniques are presented in this section. Section III presents the experimental setup and the AFM images obtained using the proposed nanopositioning system. The AFM image performances are discussed. Section IV concludes the paper.

\section{Control of The AFM NANOPOSITIONING System}

A flexure-based XYZ nanopositioner as shown in Fig. 1 was used to perform the video-rate scans in this work. The nanopositioner consists of a Z-stage mounted in series to a XY-stage. The XY-stage has a parallel-kinematic configuration. Detailed design procedures and characterization of the nanopositioner can be found in [28], [36].

\section{A. Lateral Axes}

The first resonances of the $\mathrm{X}$ and $\mathrm{Y}$ axes appear at $13.7 \mathrm{kHz}$ and $11.6 \mathrm{kHz}$ respectively. Each lateral axis is driven by a $5 \mathrm{~mm}$ $\times 5 \mathrm{~mm} \times 10 \mathrm{~mm}$ Noliac SCMAP07 piezoelectric stack actuator with a capacitance of $380 \mathrm{nF}$. The measured travel range of both axes is $7 \mu \mathrm{m}$. The fast lateral $\mathrm{X}$-axis was driven by an in-house built high-bandwidth voltage amplifier which has a peak current of $1 \mathrm{~A}$ and a voltage gain of 20. The slow Y-axis was driven by a PiezoDrive PDL200 voltage amplifier. Two 100-kHz bandwidth capacitive displacement sensors (MicroSense 6810) were used

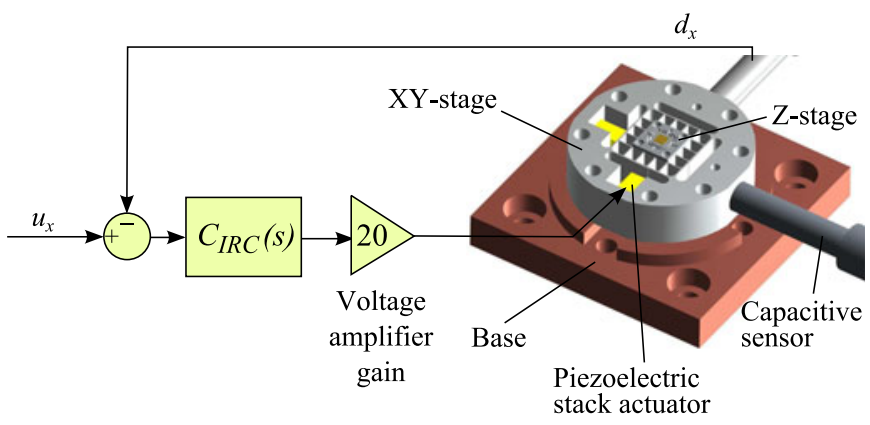

(a)
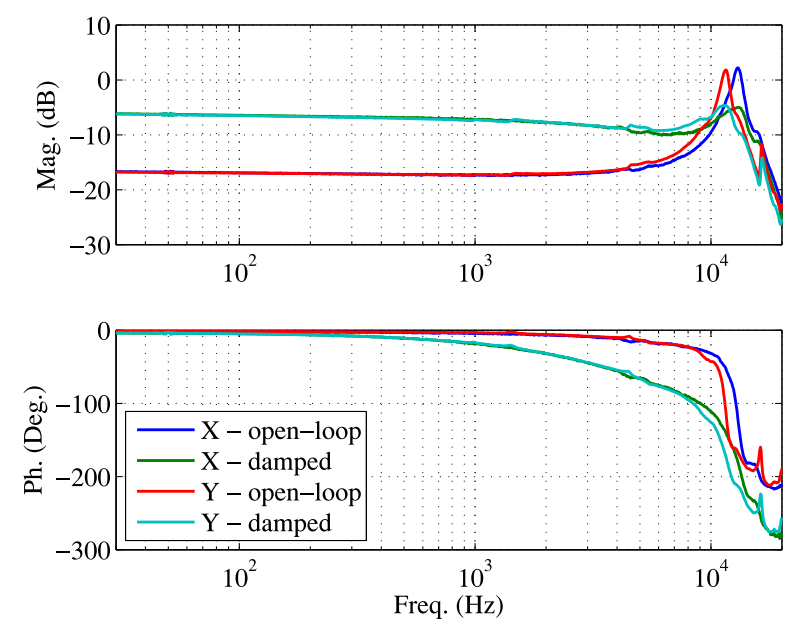

(b)

Fig. 2. (a) Block diagram of the X-axis feedback control loop. Y-axis control loop is identical to that of $\mathrm{X}$, therefore, it is not shown in the diagram. (b) $\mathrm{X}$ and $\mathrm{Y}$ axes frequency responses.

to measure the $\mathrm{X}$ and $\mathrm{Y}$ lateral motions of the nanopositioner. A Polytec Micro System Analyzer (MSA-400) was used to measure the frequency responses of the nanopositioner.

Scan-induced vibrations are a cause of common image artifacts in video-rate AFM. In this work, analog IRC were designed and implemented to damp the first resonant peaks of the two lateral axes of the nanopositioner. The block diagram of the damping control loop is shown in Fig. 2(a). Since the focus of this paper is on the vertical axis of the nanopositioner, a brief explanation of the IRC is discussed here. Further details can be found in [31], [36].

The IRC scheme includes a feed-through term and an integrator which resembles the characteristic of a low-pass filter. A common way of designing an IRC is to firstly obtain an accurate model, $G_{x / y}$ that captures the dominant resonant mode of the plant. This is followed by adding a small feed-through, $D$ to the plant so that the $G_{x / y}+D$ transfer function has zeros followed by interlaced poles, instead of the resonant poles then zeros. The modified $G_{x / y}+D$ is now more conducive to integral feedback. To select an integral gain that provides maximum damping to the system, the loop gain is analyzed. A root-locus plot shows the trajectories traveled by the poles due to the change of system gain. It also reveals the damping of each pole 

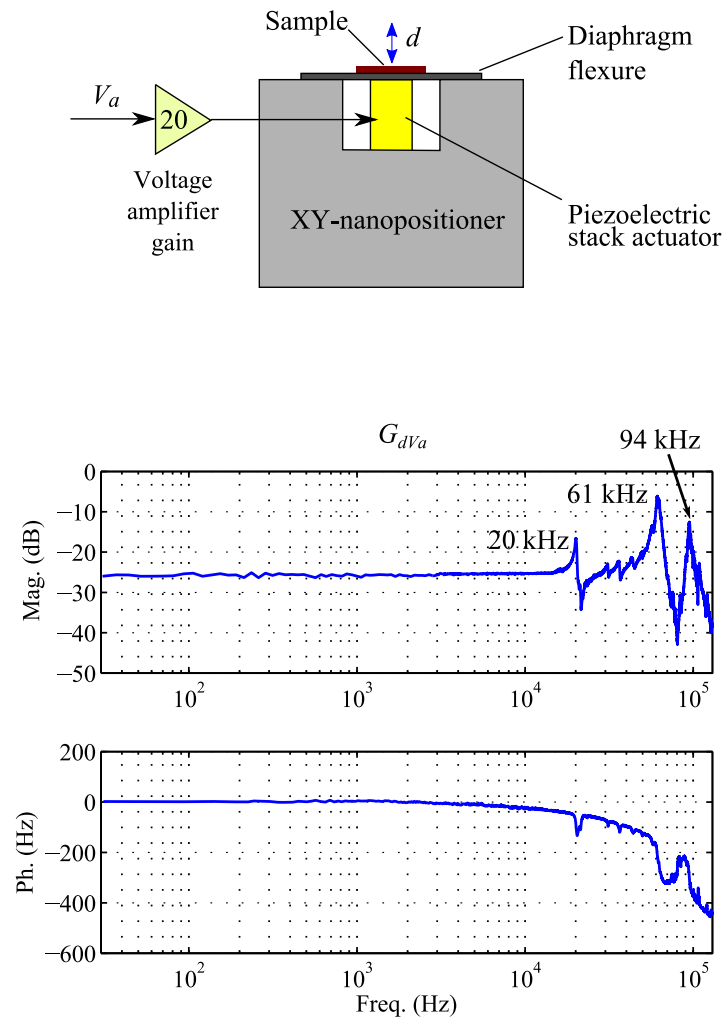

(a)
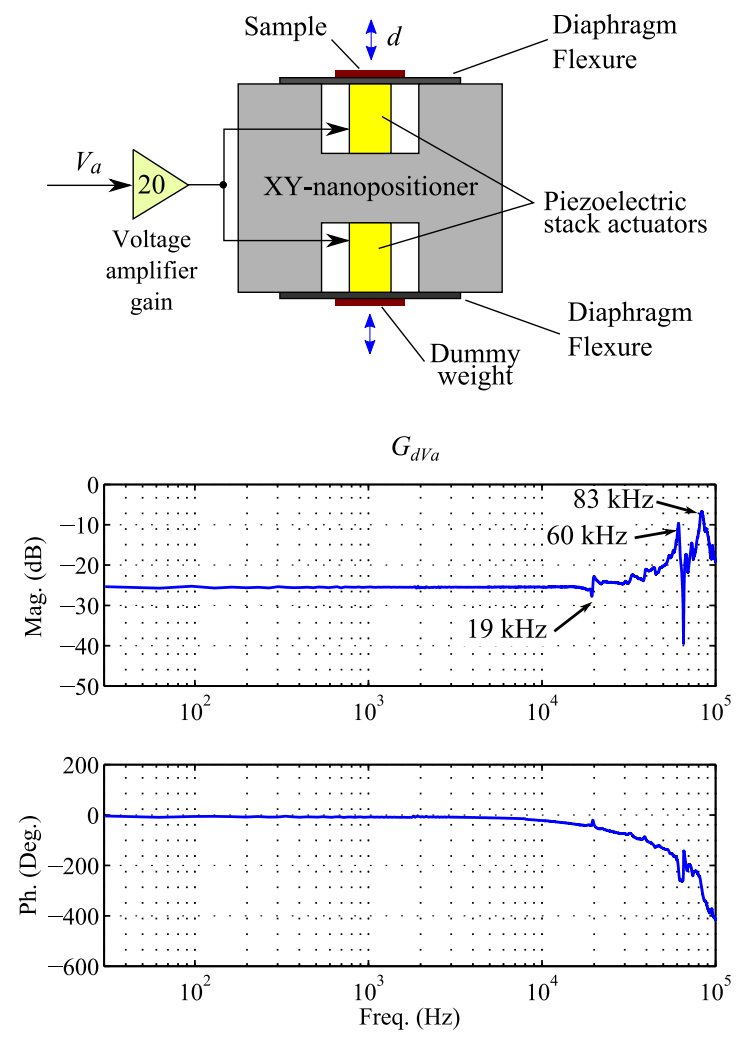

(b)

Fig. 3. Schematic and frequency response of the (a) single-mounted Z-stage without the inertial counterbalancing technique, and (b) dual-mounted Z-stage with the inertial counterbalanced damping technique.

along the trajectory. A gain with maximum damping can therefore be chosen from the root-locus plot. The transfer function of the IRC implemented on both the lateral feedback control loops is $C_{I R C}=-100000 /(s+45000)$. The two axes openand closed-loop frequency responses are shown in Fig. 2(b). The IRCs augment substantial damping to the resonances reducing magnitudes of the two resonant peaks by at least $18 \mathrm{~dB}$.

\section{B. Vertical Axis}

One approach to generate $\mathrm{Z}$ movement in the AFM system is to mount a Z-stage on the XY-stage [37], [38]. Here, this arrangement is referred to as the single-mounted $Z$-stage configuration. This approach is simple and straightforward, however, the inertial forces caused by the rapid movement of the $\mathrm{Z}$ actuator tend to excite the resonant modes of the XY-stage. To demonstrate this, a Z-stage which consists of a $3 \mathrm{~mm} \times 3 \mathrm{~mm} \times 6 \mathrm{~mm}$ piezoelectric stack actuator and a diaphragm flexure, was mounted on the XY-stage as shown in Fig. 3(a). A high-bandwidth voltage amplifier with a voltage gain of 20 was used to drive the piezoelectric stack actuator. The diaphragm flexure was used to guide the motions as well as to provide preloads to the piezoelectric stack actuator. A sample was glued onto the platform of the Z-stage. When the piezoelectric stack actuator was excited by a pseudo-random input with a bandwidth from $10 \mathrm{~Hz}$ to $100 \mathrm{kHz}$ (generated by the MSA-400), the measured first resonant peak appears at $20 \mathrm{kHz}$. This resonance is the trampoline mode of the XY-stage instead of the vertical mode of the Z-stage [28]. This is due to the impulsive forces, induced by the fast movement of the vertical piezoelectric actuator, which propagate through the structure and excite the XY-stage. Fig. 3(a) shows the measured frequency response from the input voltage $V_{a}$ to sample displacement $d$ of the single-mounted Z-stage. In this configuration, the maximum achievable bandwidth of the vertical feedback control loop is limited by the low $20-\mathrm{kHz}$ resonant peak.

1) Passive Damping With Dual-mounted Configuration: The trampoline mode of the XY-stage can be suppressed passively by mounting an identical Z-stage to the bottom of the XY-stage as illustrated in Fig. 3(b). A dummy sample, with approximately the same weight as the sample, was glued onto the platform of the bottom Z-stage. The two piezoelectric stack actuators are simultaneously actuated in opposite directions, and when the mass is balanced correctly, the bottom actuator counterbalances the inertial forces exerted by the top actuator, which in turns effectively cancels the $20 \mathrm{kHz}$ vertical resonant peak of the XY-stage. Fig. 3(b) shows the measured frequency response from $V_{a}$ to $d$ of the dual-mounted Z-stage. Note that the three resonant peaks are located at frequencies slightly different from that of the single-mounted configuration due to the weight difference between the two devices. The zero-pole pattern in the magnitude response is due to the slightly heavier dummy sample compared to that of the sample at the top Z-stage [28]. The low $20-\mathrm{kHz}$ resonant peak is eliminated in this dual-mounted 


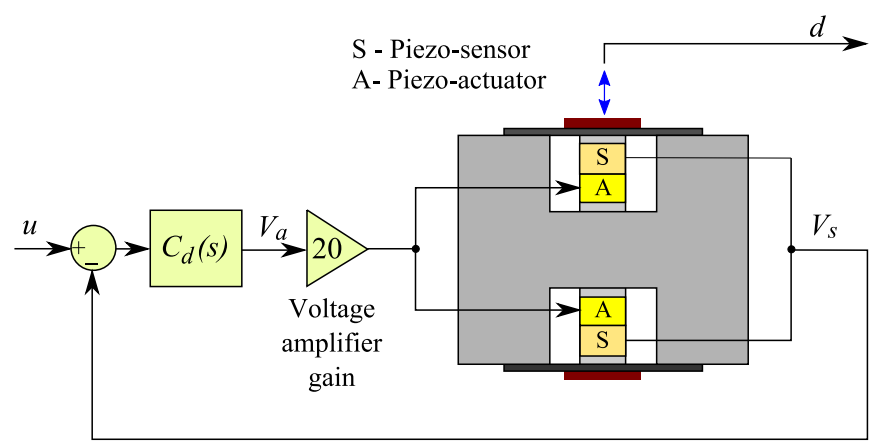

Fig. 4. Feedback control loop of the dual-mounted Z-stage driven by piezoelectric stack actuators with collocated force sensors.

configuration showing insignificant magnitude and phase profiles in the frequency response. As shown in Fig. 3(b), the dominant resonant peaks are now located at $60 \mathrm{kHz}$ and $83 \mathrm{kHz}$. A controller can then be designed to control these resonant modes in order to achieve a broader Z-bandwidth.

2) Active Damping Control: In order to control the $60 \mathrm{kHz}$ and $83 \mathrm{kHz}$ modes, the setup shown in Fig. 4 was used. The idea is to use piezoelectric sensors to generate the requisite measurement. These sensors exhibit high sensitivity, wide bandwidth and low noise at high frequencies [39]. To construct the collocated force sensor, two $3 \mathrm{~mm} \times 3 \mathrm{~mm} \times 2 \mathrm{~mm}$ Noliac CMAP06 piezoelectric plate transducers were glued together; one to serve as an actuator and the other as a force sensor. Fig. 4 shows the wiring of the Z-stage in the dual-mounted configuration and its associated control block diagram.

Active vibration control of the vertical axis may appear as quite a challenging task, due to the presence of a relatively large number of vibration modes in $G_{V_{s} V_{a}}(s)$, as illustrated in Fig. 5(a). However, the collocated nature of the actuator/sensor pairs, makes it possible to design and implement a controller that has a simple structure and is very efficient in damping those vibration modes. In particular, the frequency response function of $-G_{V_{s} V_{a}}(s)$ resembles a negative imaginary system [30]. Positive position feedback connection of a negative imaginary system, $G(s)$ with a strictly negative imaginary controller, $K(s)$ is guaranteed to be stable as long as $G(0) K(0)<1$ [35], [40].

This makes the IRC a viable compensator for $G_{V_{s} V_{a}}(s)$, in negative feedback. The IRC has been used as a damping controller in a variety of flexible structures with collocated sensors and actuators [30], [31], [35]. The controller has a simple structure, is quite robust, is easy to implement and is capable of damping a relatively large number of vibration modes of the structure.

The feedback control loop is illustrated in Fig. 6. The IRC controller $C_{d}(s)$ has the following structure,

$$
C_{d}(s)=\frac{\gamma}{s+\eta}, \quad \eta, \gamma>0 .
$$

The control problem boils down to selecting parameters $\eta$ and $\gamma$ so that adequate bandwidth is achieved and $-G_{V_{s} V_{a}}(0) C_{d}(0)<1$. The latter constraint guarantees closed loop stability when $-G_{V_{s} V_{a}}(s)$ is negative imaginary.
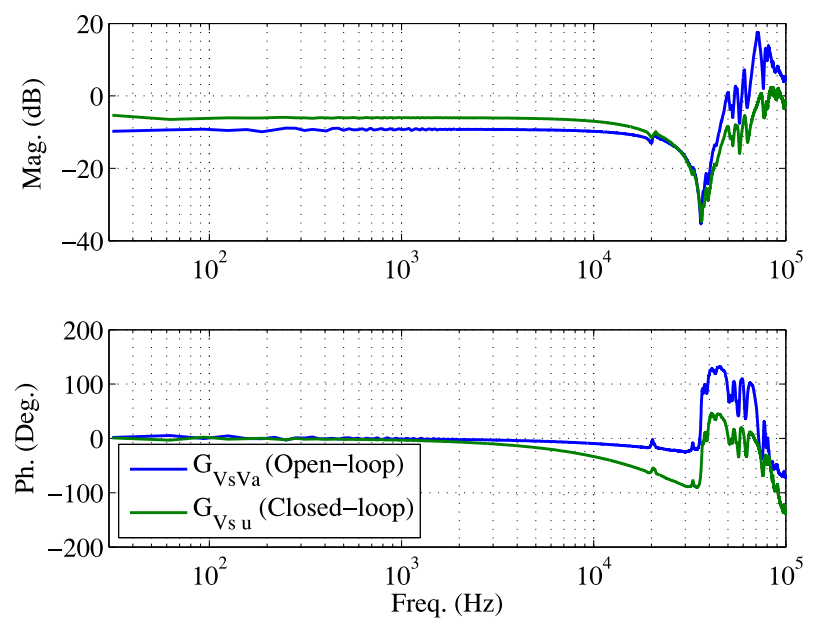

(a)
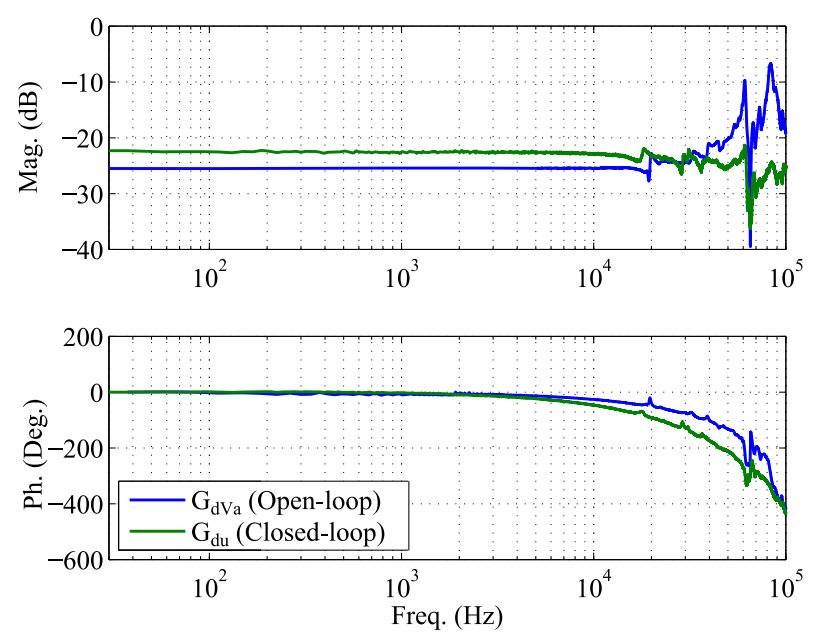

(b)

Fig. 5. Measured frequency responses of the Z-stage.

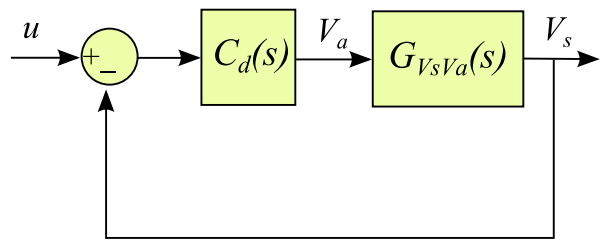

Fig. 6. The IRC loop realized with a collocated force sensor.

There are a number of ways to determine $\eta$ and $\gamma$. One method is discussed in Sec. II-A. Another tuning method can be found in [41]. The method chosen here is to first select $\eta$ so that over the range of frequencies where vibration modes of the nanopositioner exist, the controller resembles an integrator. In particular, phase of the controller must be close to $-90^{\circ}$ at resonance frequencies of the nanopositioner. Having selected a pole for the controller, all that remains is to determine an appropriate gain. A gain is selected so that the modes are suppressed by at least $15 \mathrm{~dB}$ with gain and phase margins no less than $6 \mathrm{~dB}$ and $45^{\circ}$ 


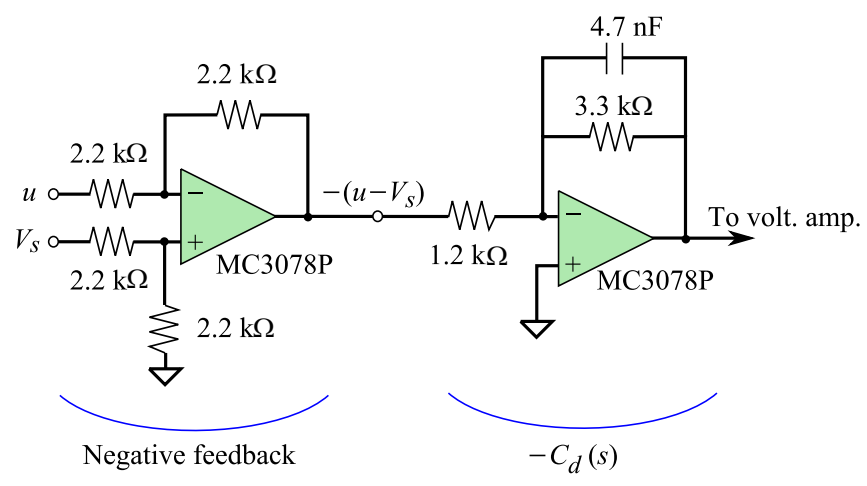

Fig. 7. Circuit diagram of the IRC control loop in the vertical axis.

respectively. Due to the high-bandwidth nature of the plant, the compensator was implemented as an analog controller.

The controller that was designed and implemented has the transfer function,

$$
C_{d}(s)=\frac{177304.96}{s+6.447 \times 10^{4}} .
$$

The corresponding circuit diagram is shown in Fig. 7. This controller achieves gain and phase margins of $9.97 \mathrm{~dB}$ and $45.7^{\circ}$ respectively. To evaluate the effectiveness of this controller, measured frequency responses from $V_{a}$ to $V_{s}$ and from $V_{a}$ to $d$ are plotted in Fig. 5. Here, $d$ is the displacement of the sample measured using a Polytec MSV-400 Laser Scanning Vibrometer. As illustrated in the measured frequency responses of $G_{V_{s} u}$ and $G_{d u}$, in Fig. 5, the $60-\mathrm{kHz}$ and $83-\mathrm{kHz}$ resonant peaks are substantially damped. From the magnitude response of $G_{d u}(s)$, it can be observed that these resonant peaks are suppressed by approximately $15.8 \mathrm{~dB}$ and $19 \mathrm{~dB}$ respectively. The nearly flat frequency response of $G_{d u}(s)$ makes the stage ideal for high-speed atomic force microscopy. To this end, we point out that although frequency response of $-G_{V_{s} V_{a}}(s)$ deviates from the negative imaginary criterion over a certain range of frequencies, the designed IRC appears to be quite robust and functions with satisfactory performance.

\section{IMAGING EXPERIMENTS AND RESULTS}

The nanopositioner whose vibratory dynamics are damped by a combination of active and passive methods is used as the scanning stage of an AFM to achieve video-rate imaging. The device was mounted under a commercial Nanosurf EasyScan 2 AFM as shown in Fig. 8(a). To operate the AFM in constant-force contact mode, the AFM optical system was used to measure the cantilever's deflections. The AFM's vertical feedback loop was replaced with an external high-gain integral feedback control loop in order to regulate the tip-sample interaction forces at highspeeds. The schematic of this arrangement is shown in Fig. 8(b). An ASM 750-HD calibration sample with a pitch of $738 \mathrm{~nm}$ and a feature height of $100 \mathrm{~nm}$ was used to evaluate the AFM images. The sample was mounted on the nanopositioner and was raster-scanned with pure sinusoidal waveforms at $100 \mathrm{~Hz}, 200$ $\mathrm{Hz}, 500 \mathrm{~Hz}$ and $1000 \mathrm{~Hz}$. Sinusoidal scanning was used to avoid vibrations induced from the lateral axes. This method is used in

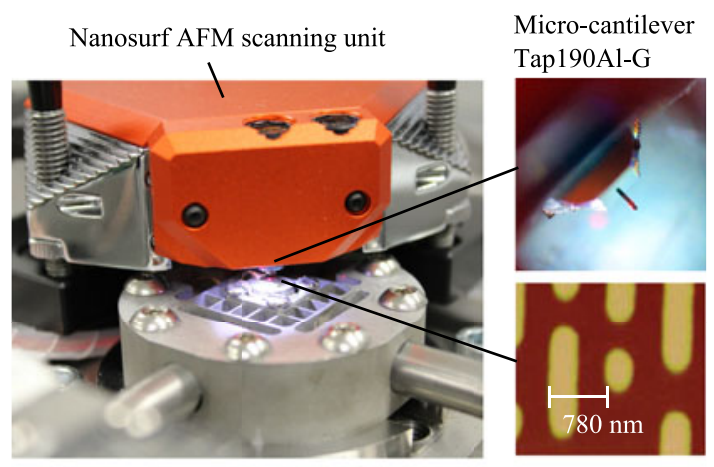

Calibration grating ASM 750-HD

(a)

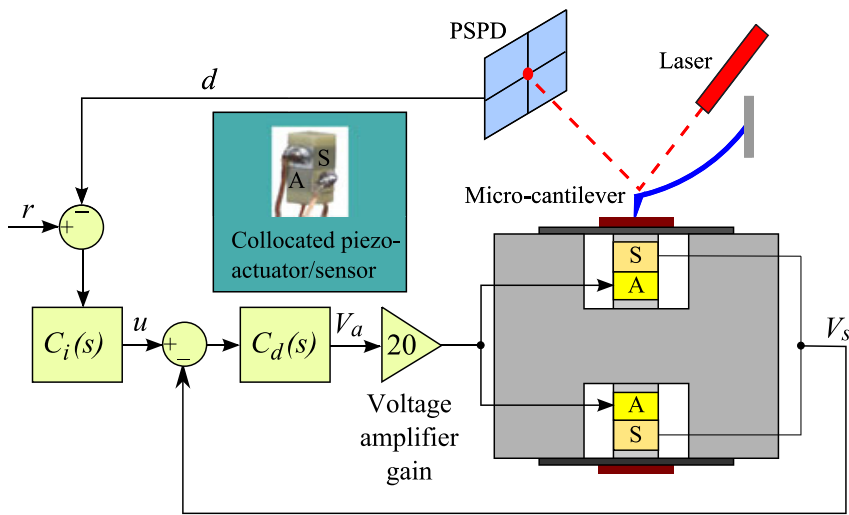

(b)

Fig. 8. (a) Nanosurf EasyScan 2 AFM and the XYZ nanopositioner. (b) Operating AFM in constant-force contact mode using the proposed control techniques.

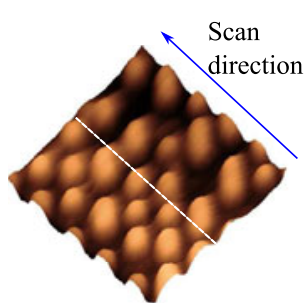

(a)

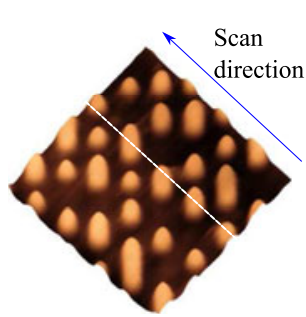

(c)

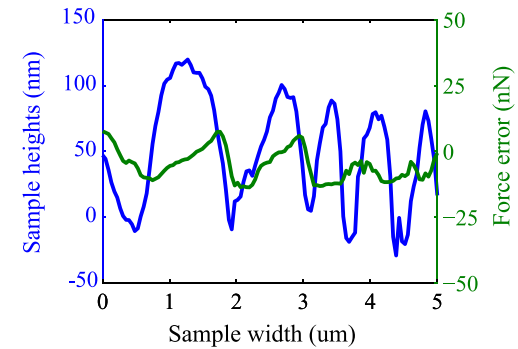

(b)

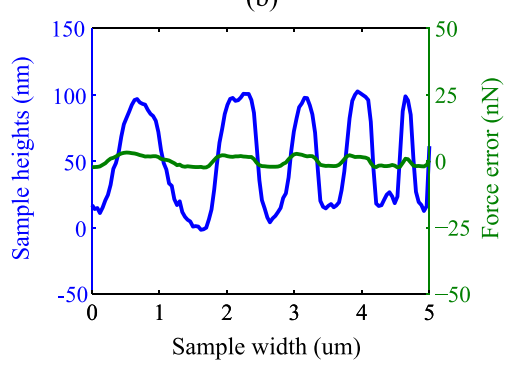

(d)
Fig. 9. Comparison of constant-force contact mode AFM images obtained at $200 \mathrm{~Hz}$ line-rate with the vertical loop bandwidth of $2.3 \mathrm{kHz}$ [(a) \& (b)] and $28.1 \mathrm{kHz}[$ (c) \& (d)]. The size of the image is $5 \mu \mathrm{m} \times 5 \mu \mathrm{m}$. (b) \& (d) show single-line information of the sample heights and force errors of the two scans. Locations of the single-line information taken from the AFM images are shown in white dashed lines in (a) \& (b). 


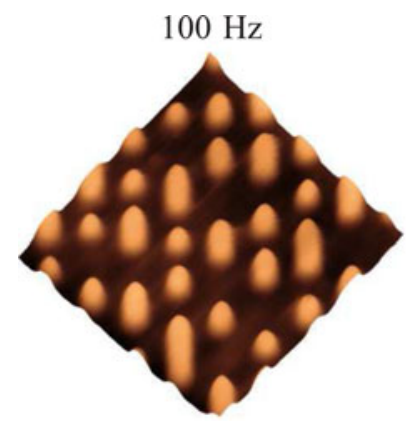

RMS force error $=5.32 \mathrm{nN}$

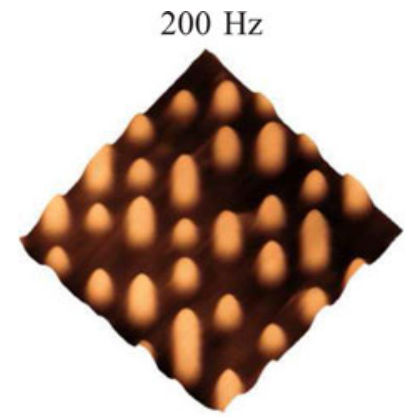

RMS force error $=6.01 \mathrm{nN}$

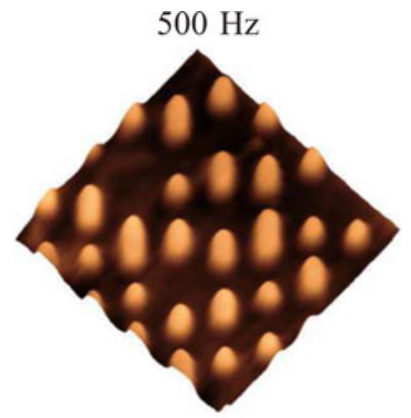

RMS force error $=6.87 \mathrm{nN}$

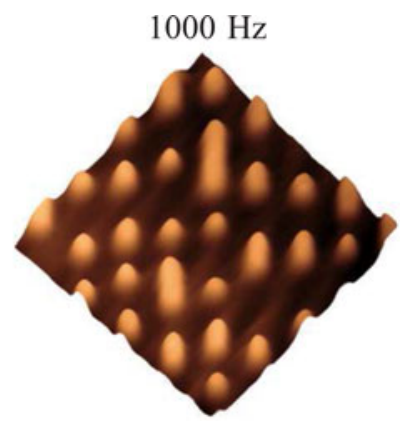

RMS force error $=8.36 \mathrm{nN}$

Fig. 10. AFM images of an $5 \mu \mathrm{m} \times 5 \mu \mathrm{m}$ area of the calibration gratings with $128 \times 128$ pixel resolution obtained at $100 \mathrm{~Hz}, 200 \mathrm{~Hz}, 500 \mathrm{~Hz}$ and $1000 \mathrm{~Hz}$ line rates respectively.

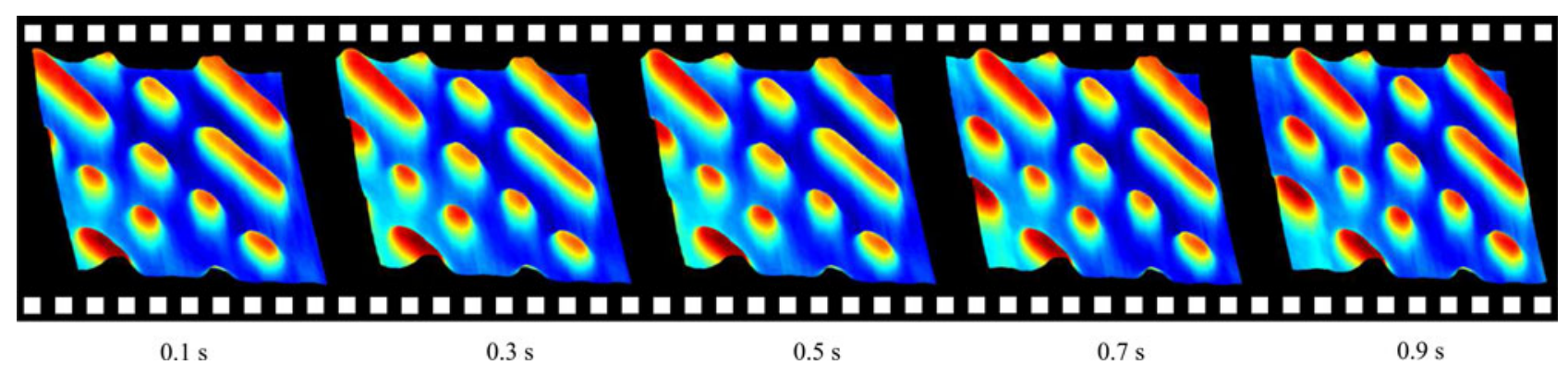

Fig. 11. Image sequence of the sample recorded at 10 frames/s showing the movement of the sample from right to left. Every second image in the sequence is illustrated. Scan size: $3.2 \mu \mathrm{m} \times 3.2 \mu \mathrm{m}$. Frame pixels: $100 \times 100$.

tuning fork based AFMs [42] and a number of other non-raster scanning techniques [22], [43]-[46]. A BudgetSensors AFM cantilever Tap190Al-G with a force constant of $48 \mathrm{~N} / \mathrm{m}$ and the first resonance frequency of $190 \mathrm{kHz}$ was used to perform the scans. The tip-sample force was regulated at $40 \mathrm{nN}$ during the scans. A MSA-050-3D Polytec data acquisition system with a maximum sampling rate of $2.56 \mathrm{MHz}$ was used to record the images.

With the vertical damping control techniques in place, we were able to increase the gain of the integral controller $C_{i}(s)$ to $1.12 \times 10^{6}$ with loop gain and phase margins of $10 \mathrm{~dB}$ and $52.4^{\circ}$ respectively. This increases the vertical tracking bandwidth from $2.3 \mathrm{kHz}$ (without damping) to $28.1 \mathrm{kHz}$. Fig. 9 compares the image quality obtained at $200 \mathrm{~Hz}$ line rate with vertical tracking bandwidths of $2.3 \mathrm{kHz}$ and $28.1 \mathrm{kHz}$ respectively. The image captured with the $2.3 \mathrm{kHz}$ bandwidth shows a "smudging" effect on the features which is an artifact associated with insufficient vertical tracking bandwidth [1], [21]. Figs. 9(b) and 9(d) show the single-line sample heights (in $\mathrm{nm}$ ) and tracking errors (in $\mathrm{nN}$ ) of the undamped and damped systems respectively. The tracking force error of the undamped system is significantly higher than that of the damped system. It is evident that the image quality is substantially improved with a tenfold increase in the vertical tracking bandwidth.

Fig. 10 shows images of a $5 \mu \mathrm{m} \times 5 \mu \mathrm{m}$ area of the sample grating recorded at the four line rates. A sequence of images showing the movement of the sample (from right to left) recorded at 10 frames/s is shown in Fig. 11. All images show similar quality with their RMS force errors kept within $8.36 \mathrm{nN}$. These results cannot be achieved by using the commercial AFM scanner and its associated vertical feedback control loop.

\section{CONCLUSIONS}

One of the main limitations in obtaining high-quality videorate AFM images in constant-force contact mode is the insufficient bandwidth of the vertical feedback control loop. In this work, this hurdle was overcame by combining a high-speed flexure-based nanopositioner with active and passive damping control techniques. To use passive damping control, two identical Z-stages were mounted to the XY-stage. The Z piezoelectric stack actuators were actuated concurrently with the same magnitude but in opposite directions. This dual-mounted configuration counterbalances the impulsive force induced by the top actuator, which in turns, eliminates the low $20-\mathrm{kHz}$ resonant peak of the nanopositioner. With the $20-\mathrm{kHz}$ resonance suppressed, an active controller was implemented to suppress the subsequent resonant peaks that live at $60 \mathrm{kHz}$ and above. IRC technique was used to control these resonances. With the combination of the above mentioned damping techniques, the vertical feedback bandwidth was increased to $28.1 \mathrm{kHz}$ which is a tenfold improvement on the open-loop nanopositioning system. This high-bandwidth nanopositioning system enabled us to obtain high-quality constant-force video-rate AFM images at 
10 frames/s without artifacts related to scan-induced vibrations and insufficient vertical tracking bandwidth.

\section{REFERENCES}

[1] Y. K. Yong, S. O. R. Moheimani, B. J. Kenton, and K. K. Leang, "Invited review article: High-speed flexure-guided nanopositioning: Mechanical design and control issues," Rev. Sci. Instrum., vol. 83, no. 12, art. no. $121101,2012$.

[2] D. Abramovitch, S. Andersson, L. Pao, and G. Schitter, "A tutorial on the mechanisms, dynamics, and control of atomic force microscopes," in Proc. Amer. Control Conf., Jul. 2007, pp. 3488-3502.

[3] G. Binnig, C. F. Quate, and C. Gerber, "Atomic force microscope," Phys. Rev. Lett., vol. 56, pp. 930-933, 1986.

[4] H. Watanabe, T. Uchihashi, T. Kobashi, M. Shibata, J. Nishiyama, R. Yasuda, and T. Ando, "Wide-area scanner for high-speed atomic force microscopy," Rev. Sci. Instrum., vol. 84, no. 5, art. no. 053 702, 2013.

[5] T. Ando, "High-speed atomic force microscopy coming of age," Nanotechnology, vol. 23, no. 6, art. no. 062001, 2012.

[6] P. K. Hansma, G. Schitter, G. E. Fantner, and C. Prate, "High-speed atomic force microscopy," Science, vol. 314, no. 3799, pp. 601-602, 2006.

[7] O. A. Ageev, N. I. Alyabieva, B. G. Konoplev, V. Smirnov, and V. V. Tkachuk, "Investigation of the nanodiagnostics probe modes for semiconductor resistivity measurements by atomic force microscopy," Adv. Mater. Res., vol. 894, pp. 374-378, 2014.

[8] J. Foucher, N. Rana, and C. Dezauzier, "3D-AFM enhancement for CD metrology dedicated to lithography sub-28-nm node requirements," Proc. SPIE, vol. 7638, art. no. 763802, 2010.

[9] R. A. Oliver, "Advances in AFM for the electrical characterization of semiconductors," Reports Progress Phys., vol. 71, no. 7, art. no. 076501, 2008.

[10] S. Miyake, M. Wang, and J. Kim, "Silicon nanofabrication by atomic force microscopy-based mechanical processing," J. Nanotechnol., vol. 2014, art. no. 102404, 2014.

[11] A. A. Tseng, "Advancements and challenges in development of atomic force microscopy for nanofabrication," Nano Today, vol. 6, no. 5, pp. 493-509, 2011.

[12] M. S. Johannes, D. G. Cole, and R. L. Clark, "Atomic force microscope based nanofabrication of master pattern molds for use in soft lithography," Appl. Phys. Lett., vol. 91, no. 12, art. no. 123 111, 2007.

[13] G. Binnig, M. Despont, U. Drechsler, W. Haberle, M. Lutwyche, P. Vettiger, H. Mamin, B. Chui, and T. Kenny, "Ultrahigh-density atomic force microscopy data storage with erase capability," Appl. Phys. Lett., vol. 74, no. 9, pp. 1329-1331, 1999.

[14] P. Vettiger, G. Cross, M. Despont, U. Drechsler, U. Dürig, B. Gotsmann, W. Häberle, M. A. Lantz, H. E. Rothuizen, R. Stutz, and G. K. Binnig, "The "Milipede"-Nanotechnology entering data storage," IEEE Trans. Nanotechnol., vol. 1, no. 1, pp. 39-55, Mar. 2002.

[15] B. Bhushan, Springer Handbook of Nanotechnology. New York, NY, USA: Springer, 2004.

[16] J. A. Vicary and M. J. Miles, "Real-time nanofabrication with high-speed atomic force microscopy," Nanotechnology, vol. 20, no. 9, art. no. 095302 , 2009.

[17] G. Schitter and M. J. Rost, "Scanning probe microscopy at video-rate," Mater. Today, vol. 11, pp. 40-48, 2008.

[18] Y. K. Yong and S. O. R. Moheimani, "A compact XYZ scanner for fast atomic force microscopy in constant force contact mode," in Proc. IEEE Int. Conf. Adv. Intell. Mechatron., pp. 225-230, Jul. 2010.

[19] G. E. Fantner, G. Schitter, J. H. Kindt, T. Ivanov, K. Ivanova, R. Patel, N. Holten-Andersen, J. Adams, P. J. Thurner, I. W. Rangelow, and P. K. Hansma, "Components for high speed atomic force microscopy," Ultramicroscopy, vol. 106, pp. 881-887, 2006.

[20] G. Schitter, K. J. Åstrom, B. DeMartini, G. E. Fantner, K. Turner, P. J. Thurner, and P. K. Hansma, "Design and modeling of a high-speed scanner for atomic force microscopy," in Proc. Amer. Control Conf., Jun. 2006, pp. 502-507.

[21] A. J. Fleming, B. J. Kenton, and K. K. Leang, "Bridging the gap between conventional and video-speed scanning probe microscopes," Ultramicroscopy, vol. 110, no. 9, pp. 1205-1214, 2010.

[22] Y. K. Yong, A. Bazaei, and S. O. R. Moheimani, "Video-rate Lissajousscan atomic force microscopy," IEEE Trans. Nanotechnol., vol. 13, no. 1, pp. 85-93, Jan. 2014.
[23] B. J. Kenton and K. K. Leang, "Design and control of a three-axis serialkinematic high-bandwidth nanopositioner," IEEE Trans. Mechatron., vol. 17, no. 2, pp. 356-368, Apr. 2012.

[24] A. D. L. Humphris, M. J. Miles, and J. K. Hobbs, "A mechanical microscope: High-speed atomic force microscopy," Appl. Phys. Lett., vol. 86, no. 3, art. no. 034 106, 2005.

[25] J. Kwon, J. Hong, Y.-S. Kim, D.-Y. Lee, K. Lee, S.-M. Lee, and S.-I. Park, "Atomic force microscope with improved scan accuracy, scan speed, and optical vision," Rev. Sci. Instrum., vol. 74, no. 10, pp. 4378-4383, 2003.

[26] A. Fleming, "Dual-stage vertical feedback for high-speed scanning probe microscopy," IEEE Trans. Control Syst. Technol., vol. 19, no. 1, pp. 156-165, Jan. 2011.

[27] B. J. Kenton, A. J. Fleming, and K. K. Leang, "Compact ultra-fast vertical nanopositioner for improving scanning probe microscope scan speed," Rev. Sci. Instrum., vol. 82, no. 12, art. no. 123 703, 2011.

[28] Y. K. Yong and S. O. R. Moheimani, "Design of an inertially counterbalanced Z-nanopositioner for high-speed atomic force microscopy," IEEE Trans. Nanotechnol., vol. 12, no. 2, pp. 137-145, Mar. 2013.

[29] T. Ando, T. Uchihashi, N. Kodera, D. Yamamoto, A. Miyagi, M. Taniguchi, and H. Yamashita, "High-speed AFM and nano-visualization of biomolecular processes," Pflügers Archiv Eur. J. Physiology, vol. 456, no. 1, pp. 211-225, 2008.

[30] I. Petersen and A. Lanzon, "Feedback control of negative-imaginary systems," IEEE Control Syst. Mag., vol. 30, no. 5, pp. 54-72, Oct. 2010.

[31] S. S. Aphale, A. J. Fleming, and S. O. R. Moheimani, "Integral resonant control of collocated smart structures," Smart Mater. Struct., vol. 16, pp. 439-446, 2007.

[32] A. Preumont, B. de Marneffe, A. Deraemaeker, and F. Bossens, "The damping of a truss structure with a piezoelectric transducer," Comput. Struct., vol. 86, no. 3-5, pp. 227-239, 2008.

[33] A. Preumont, Vibration Control of Active Structures: An Introduction. New York, NY, USA: Springer, 2011.

[34] A. J. Fleming, "Nanopositioning system with force feedback for highperformance tracking and vibration control," IEEE Trans. Mechatron., vol. 15, no. 3, pp. 433-447, Jun. 2010.

[35] B. Bhikkaji and S. O. R. Moheimani, "Integral resonant control of a piezoelectric tube actuator for fast nano-scale positioning," IEEE Trans. Mechatron., vol. 13, no. 5, pp. 530-537, Oct. 2008.

[36] Y. K. Yong, B. Bhikkaji, and S. O. R. Moheimani, "Design, modeling and FPAA-based control of a high-speed atomic force microscope nanopositioner," IEEE Trans. Mechatron., vol. 18, no. 3, pp. 1060-1071, Jun. 2013.

[37] G. Schitter, K. J. Åstrom, B. DeMartini, P. J. Thurner, K. L. Turner, and P. K. Hansma, "Design and modeling of a high-speed AFM-scanner," IEEE Trans. Control Syst. Technol., vol. 15, no. 5, pp. 906-915, Sep. 2007.

[38] Y. K. Yong, B. Bhikkaji, and S. O. R. Moheimani, "Analog control of a high-speed atomic force microscope scanner," in Proc. IEEE Int. Conf. Adv. Intell. Mechatron., pp. 646-651, Jul. 2011.

[39] Y. K. Yong, A. Fleming, and S. O. R. Moheimani, "A novel piezoelectric strain sensor for simultaneous damping and tracking control of a high-speed nanopositioner," IEEE Trans. Mechatron., vol. 18, no. 3, pp. 1113-1121, Jun. 2013.

[40] B. Bhikkaji, S. O. R. Moheimani, and I. R. Petersen, "A negative imaginary approach to modeling and control of a collocated structure," IEEE Trans. Mechatron., vol. 17, no. 4, pp. 717-727, Aug. 2012.

[41] A. A. Eielsen, M. Burger, J. T. Gravdahl, and K. Y. Pettersen, "PI2-controller applied to a piezoelectric nanopositioner using conditional integrators and optimal tuning," in Proc. IFAC World Congr., pp. 887-892, Aug. 2011.

[42] L. M. Picco, L. Bozec, A. Ulcinas, D. J. Engledew, M. Antognozzi, M. A. Horton, and M. J. Miles, "Breaking the speed limit with atomic force microscopy," Nanotechnology, vol. 18, art. no. 044 030, 2007.

[43] I. A. Mahmood, S. O. R. Moheimani, and B. Bhikkaji, "A new scanning method for fast atomic force microscopy," IEEE Trans. Nanotechnol., vol. 10, no. 2, pp. 203-216, Mar. 2011.

[44] Y. K. Yong, S. O. R. Moheimani, and I. R. Petersen, "High-speed cycloid-scan atomic force microscopy," Nanotechnology, vol. 21, no. 36, art. no. 365 503, 2010.

[45] A. Bazaei, Y. K. Yong, and S. O. R. Moheimani, "High-speed Lissajousscan atomic force microscopy: Scan pattern planning and control design issues," Rev. Sci. Instrum., vol. 83, no. 6, art. no. 063701 , 2012. 
[46] T. Tuma, J. Lygeros, V. Kartik, A. Sebastian, and A. Pantazi, "Highspeed multiresolution scanning probe microscopy based on Lissajous scan trajectories," Nanotechnology, vol. 23, no. 18, art. no. 185 501, 2012.

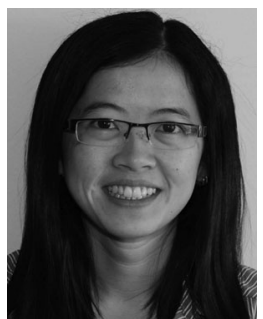

Yuen Kuan Yong (M'09) received the B. Eng. degree in mechatronic engineering and the Ph.D. degree in mechanical engineering from the University of Adelaide, Adelaide, Australia, in 2001 and 2007, respectively.

She is currently an Australian Research Council Discovery Early Career Researcher Award Fellow at the University of Newcastle, Callaghan, Australia. Her research interests include the design and control of nanopositioning systems, high-speed atomic force microscopy, finite-element analysis of smart materials and structures, sensing and actuation, and robot insects. She is also an Associate Editor of the International Journal of Advanced Robotic Systems.

Dr. Yong's research has been recognized by a number of awards, including the 2008 IEEE International Conference on Advanced Intelligent Mechatronics Best Conference Paper Finalist Award, the University of Newcastle ViceChancellor's Awards For Research Excellence and the Pro Vice-Chancellor's Award for Excellence in Research Performance. She is a Technical Program Committee Member of the IEEE Multiconference on Systems and Control, and the International Conference on Manipulation, Manufacturing, and Measurement on the Nanoscale.

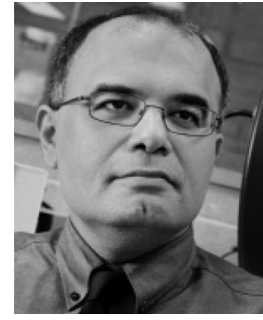

S. O. Reza Moheimani (M'96-SM'00-F'11) received the Doctoral degree from the University of New South Wales, Canberra, Australia, in 1996.

He joined the University of Newcastle in 1997 embarking on a new research program addressing the dynamics and control design issues related to highprecision mechatronic systems. He is the Founder and Director of the Laboratory for Dynamics and Control of Nanosystems, a multimillion-dollar state-of-theart research facility. He has published more than 300 refereed papers and five books and edited volumes. His current research interests include ultra-high-precision mechatronic systems, with particular emphasis on dynamics and control at the nanometer scale, including applications of control and estimation in nanopositioning systems for highspeed scanning probe microscopy, modeling and control of microcantileverbased devices, control of microactuators in microelectromechanical systems, and design, modeling and control of micromachined nanopositioners for onchip atomic force microscopy.

Dr. Moheimani is a Fellow of IFAC and the Institute of Physics, U.K. His research has been recognized with a number of awards, including the IFAC Nathaniel B. Nichols Medal in 2014, the IFAC Mechatronic Systems Award in 2013, the IEEE Control Systems Technology Award in 2009, the Australian Research Council Future Fellowship in 2009, the IEEE Transactions on Control Systems Technology Outstanding Paper Award in 2007, the Australian Research Council Post Doctoral Fellowship in 1999, and several best student paper awards in various conferences. He has served on the editorial boards of a number of journals, including the IEEE TRANSACTIONS ON MECHATRONICS, the IEEE TRANSACtions on CONTROL Systems TeChNOLOGY, and Control Engineering Practice. He currently chairs the IFAC Technical Committee on Mechatronic Systems, and has chaired several international conferences and workshops. 